Received: March 27, 2017

Accepted: April 03, 2017

Published: April 11, 2017

\title{
Pseudohypoaldosteronism in Two Omani Siblings with a Novel Mutation in the SCNN1A Gene
}

\author{
Hala A.J. Al-Shaikh* \\ Senior Consultant Pediatric Endocrinologist, Muscat Private Hospital, P.O. Box 79, Code \\ 133 alkhuwair, Oman.
}

*Corresponding author:Dr. Hala A.J. Al-Shaikh, Senior Consultant Pediatric Endocrinologist, Muscat Private Hospital, P.O. Box 79, Code 133 alkhuwair, Oman ; E-mail: hal_doc@yahoo.com

\section{Abstract}

\subsection{Background}

Type1 PHA is a rare heterogeneous group of disorders with resistance to the action of Aldosterone. Biochemically, it is characterized by hyponatremia, hyperkalemia, metabolic acidosis and elevated serum Aldosterone and Renin. The systemic type is caused by a defect in the epithelial sodium channel causing retention of potassium and loss of sodium in the sweat, saliva, urine and stool. Presentation is usually in the neonatal period. It has an autosomal recessive mode of inheritance and is caused by mutations in one of the ENaC subunit genes (SCNN1A, SCNN1B, SCNN1C).

\subsection{Objective}

To demonstrate the mode of presentation, clinical course, challenges and treatment of systemic PHA Type 1 in two Omani siblings from the region of Dhofar .

\subsection{Method}

The clinical notes and lab results were obtained from the computerised medical records at the treating hospitals. Genetic analysis for the second sibling was carried out at the centogene Labs in Germany.

\subsection{Results}

Both siblings presented in the first week of life. The first sibling had a more severe clinical course with skin manifestations. The clinical course was complicated by recurrent episodes of severe chest infections and electrolyte imbalance. Feeding difficulty and treatment of the electrolyte imbalance were challenges to the treating physician.
Genetic analysis for the second sibling confirmed a novel mutation in the (SCNN1A) subunit gene of the (ENaC),

\subsection{Conclusion}

Systemic Type 1 PHA presents as a neonatal emergency and is one of the differential diagnoses of neonatal hyperkalemia and hyponatremia. It can very well be confused with Congenital Adrenal Hyperplasia. The severity and extent of manifestations may differ within the same family. The disease can get milder with advancing age. Genomic testing for the carrier state in the asymptomatic siblings is recommended as well as in the parents to detect homozygosity or compound heterozygosity.

\section{Introduction}

Pseudohypoaldosteronism results from resistance to the hormone Aldosterone responsible for the regulation of sodium and potassium homeostasis. Its main site of action is the epithelial cells of the renal collecting ducts. By binding to the intracytoplasmic mineralocorticoid receptor (MR), it acts as a transcription factor. Transcription of signaling factors result in the accumulation of ENaC at the plasma membrane, promoting sodium transport into the epithelial cells. Sodium is then removed at the baso-lateral membrane of the cell by the sodium-potassium ATP ase [1]. Genetic mutations in the NR3C2 gene that encodes the mineralocorticoid receptor (MR) result in autosomal dominant renal Pseudohypoaldosteronism [2,3], while mutations in one of the ENaC subunit genes (SCNN1A, SCNN1B, SCNN1C) cause more severe autosomal recessive systemic Pseudohypoaldosteronism, [4,5]. The clinical presentation of Pseudohypoaldosteronism varies from the mere elevation of Plasma Renin Activity and Aldosterone to severe salt losing crisis with hypotensive shock [6]. In this report the initial presentation, clinical course, challenges and treatment of two Omani siblings from the region of Dhofar with systemic pseudohypoaldosteronism will be described. 


\section{Case Report}

The index case is currently 11 years old, Omani male, a product of consanguineous marriage, born at term by SVD to a primigravida. Birth weight was $3.2 \mathrm{~kg}$ and APGARS 7 and 9 at 1 and 5 minutes consecutively. There was thin stained meconium liquor and he was kept in the SCBU for observation with an uneventful stay. He was discharged on day 4 and was feeding well. He presented to the Emergency Department on day 6 of age with fever, vomiting, loose motion and excessive sweating of one day duration. On admission he was in a state of shock with severe dehydration, cyanosis, bradycardia and unrecordable blood pressure. He was resuscitated with ET intubation, IPPV, Adrenaline and cardiac massage along with repeated boluses of Normal Saline and Sodium Bicarb. Cardiac monitoring showed prolonged QRS complexes with peaked $\mathrm{T}$ waves. Investigations revealed hyperkalemia, hyponatremia with metabolic acidosis. Ca Gluconate, Sodium Resonium and Nebulised Salbutamol were added to the treatment regime, Congenital Adrenal Hyperplasia was anticipated and Cortisol and Fludrocortisone were given. Ampicillin and Cefotaxime were started after sending, blood, urine and CSF for culture to rule out sepsis. He was extubated after 3 days and discharged home on Sodium Bicarb, Sodium Chloride, Cortisol and Fludrocortisone.

Laboratory work up can be seen on Table 1. 17OHP, Cortisol and ACTH were normal excluding $\mathrm{CAH}, 21 \mathrm{OH}$ lase deficiency and congenital adrenal hypoplasia. An abdominal U/S excluded urinary system obstruction or malformation (Table 1).A high serum level of Aldosterone and Plasma Renin Activity were consistent with the diagnosis of pseudohypoaldosteronism, Cortisol and Fludrocortisone were stopped and he was kept on oral Sodium Chloride $3 \%$ at $20 \mathrm{ml}$ QID, Sodium Bicarb at $1 \mathrm{mmol} / \mathrm{kg} /$ day QID, and rectal sodium Resonium was added at $4 \mathrm{~g}$ TID. A sweat test was done at one year of age which came positive supporting a systemic type of pseudohypoaldosteronism. Serum electrolytes normalized. Sodium Bicarbwas stopped after 3 months and the electrolytes continued to be normal. He had normal growth and development.

Table 1 Initial Laboratory Investigation Results

\begin{tabular}{|l|l|}
\hline Serum Sodium & $110 \mathrm{mmol} / \mathrm{L}$ \\
\hline Serum Potassium & $8.9 \mathrm{mmol} / \mathrm{L}$ \\
\hline Urinary Sodium & $65 \mathrm{mmol} / \mathrm{L}$ \\
\hline Urinary Potassium & $2 \mathrm{mmol} / \mathrm{L}$ \\
\hline Cortisol & $417 \mathrm{nmol} / \mathrm{L}$ \\
\hline DHEA-S & $0.1 \mathrm{umol} / \mathrm{L}$ \\
\hline Testosterone & $20.4 \mathrm{nmol} / \mathrm{L}$ \\
\hline Serum Aldosterone & $3300 \mathrm{pmol} / \mathrm{L}$ \\
\hline Plasma Renin Activity & $43.1 \mathrm{ng} / \mathrm{ml} / \mathrm{hr}$ \\
\hline 17-OHP & $3.3 \mathrm{nmol} / \mathrm{L}$ \\
\hline
\end{tabular}

Urinary Steroid profile showed no evidence of Congenital Adrenal Hyper/Hypoplasia.

Urine and CSF C/S were normal. Blood C/S showed E coli and Klebsiella sensitive to Imipenem and Meropenem.

In the first year of life, the patient had recurrent episodes of chest infections associated with electrolyte imbalance, hyponatremia, hyperkalemia, and metabolic acidosis, which required admission to hospital and was treated with Sodium Resonium, Salbutamol nebulisation, Sodium Chloride, Sodium Bicarband antibiotics. Another problem faced in the management was feeding difficulty because of nausea and frequent vomiting which was treated with Naso-Gastric tube feeds and skin eczema due to the sodium loss from the skin, treated with moisturising creams. There was a notable decrease in the frequency of admissions as he grew up, with no further admissions after 7 years of age.

Currently he is doing fine with normal growth and development and good school performance. He is on Sodium Resonium, $5 \mathrm{~g}$ BID and 3\% Sodium Chloride $25 \mathrm{ml}$ BID.

Case two is the sister of the index case. She is currently one year old. She was born at 39wks of gestation by normal vaginal delivery with a birth weight of $3.2 \mathrm{~kg}$ and apgars of 8 and 10 . She presented to the Emergency Department on day 7 of life with poor feeding, vomiting, and lethargy with severe dehydration. On blood analysis she was found to have severe serum hyperkalemia of $9 \mathrm{mmol} / \mathrm{L}$,hyponatremia of $108 \mathrm{mmol} / \mathrm{L}$ and metabolic acidosis. Serum Cortisol: 437nmol/L, Aldosterone:2750 pmol/L, Plasma Renin Activity: > 184.3miu/L. The diagnosis of systemic pseudohypoaldosteronism was made. She was treated with parenteral Sodium chloride and Sodium Bicarb, nebulized Salbutamol and rectal Sodium Resonium. Her clinical course was milder than her sibling's and she was discharged on day 10 of life on oral Sodium chloride $4 \mathrm{ml}$ QID, oral Sodium Bicarb1 $\mathrm{ml}$ QID and rectal Sodium Resonium $9 \mathrm{~g}$ per day. During the first five months of life she had recurrent admissions with vomiting precipitated by upper respiratory tract infections. These episodes were associated with electrolyte imbalance and metabolic acidosis. She was treated with parenteral Sodium Chloride, Sodium Bicarb, and rectal Sodium Resonium and nebulized Salbutamol. She was clinically much better in the second half of the year and did not require any admission. She did not have any skin manifestations.

Genetic analysis was done for case two at Cento Gene Labs in Germany and she was found to have a novel mutation in the (SCNN1A) subunit gene of the (ENaC), c.385 G > A (p. Gly 129 Ser).To date this variant is not described in the Exome Aggregation Consortium.

Currently she is thriving well with Ht and Wt on the $25^{\text {th }}$ centile and attaining normal developmental milestones. She is on oral Sodium Chloride, $24 \mathrm{ml}$ QID and oral Sodium Resonium 9grams per day.

The above two siblings come from a family of consanguineous marriage. They have4 children, two affected and the other two are a symptomatic. Parental genetic analysis and the siblings' carrier state were not tested (Figure 1). 


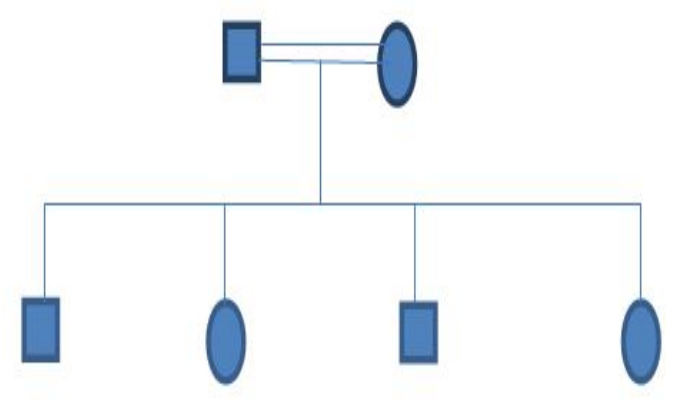

Figure 1: 11yrs old, affected 9yrs old, normal 4 yrs old, normal 1yr old, affected

\section{Discussion}

Systemic Pseudohypoaldosteronism is a disorder of resistance to Aldosterone affecting multiple organs; the kidney, the colon, the sweat and salivary glands [7]. It presents early in life with salt losing crisis, hyperkalaemia and metabolic acidosis. It can be confused with Congenital Adrenal Hyperplaisia, 21 Hydroxylase deficiency, as in our index case, and other reported cases in the literature, [8,9]. Serum Cortisol, 17OHP, ACTH, Aldosterone and Plasma Renin Activity help to differentiate between the two conditions. Other differential diagnosis include Congenital Adrenal Hypolasia and renal infection or obstruction. Treatment with Hydrocortisone, Fludrocortisone, and Sodium supplements should be started until the laboratory results are obtained, as it can be lifesaving in cases of CAH.

Over twenty genetic mutations causing Systemic Pseudohypoaldosteronism have been detected so far $[10,11]$. The mutations are in one of the three subunits (SCNN1A, SCNN1B and SCNN1C) of the ENaC responsible for Sodium transport, with the majority affecting the exon 8 of the SCNN1A gene. No strong genotype/phenotype correlation has yet been established due to the rarity of this condition. However, non-missence mutations resulting in a truncated protein are associated with a more severe disease course $[10,11]$. In our second case, a novel mutation, c. $385 \mathrm{G}>\mathrm{A}$ (p. Gly 129 Ser) in the gene encoding the SCNN1A has been detected, the significance of which is yet uncertain. Genetic analysis was not performed on the index case. The second case had a milder clinical course with fewer episodes of respiratory infections requiring hospital admissions as compared with her elder affected sibling. Assuming that the two siblings have the same mutation, it can be seen that phenotypic variations could exist within the same genotypic mutation. Genomic testing of the index case, testing for the carrier state in the asymptomatic siblings as well as in the parents to detect homozygosityor compound heterozygosity is highly recommended especially in a community with a high rate of consanguineous marriage [12 ].

The treatment of Systemic Pseudohypoaldosteronism constitutes a challenge to the treating physician. The standard treatment is adequate rehydration, replacement of salt loss with Sodium Chloride supplements(3-30 meq/kg/day), and correction of hyperkalemia with potassium exchange resins; Sodium polysterene sulphonate $(2-3 \mathrm{~g} / \mathrm{kg} /$ day) with a low potassium diet [13].The disease course is interrupted by recurrent episodes of salt losing crisis with hyperkalemia associated with nausea, vomiting and feeding difficulty as well as a chronic pulmonary syndrome associated with or without chest infections, [13]. The repeated chest symptoms result from accumulation of fluid and sodium in the airways as a result of defective absorption from the affected epithelial sodium channels [13,14]. This could be confused with Cystic Fibrosis, especially with a positive Sweat Chloride Test. Feeding difficulties might require fundoplication or gastrostomy feeds, and chest infections usually need hospitilisation to treat the infection and the electrolyte imbalance associated with it [6]. Some patients have skin manifestations in the form of miliariarubra-like cutaneous eruptions and skin infections [15] resulting from blockage and inflammation of the exocrine sweat glands due to high-sweat sodium concentrations [16]. Eye manifestations occur in the form of projections over the eyelid margins representing enlarged meibomian glands [16, 17]. Other clinical presentations include polyhydramnios [18], neonatal respiratory distress syndrome, cholelithiasis and cardiac arrythmias $[6,9,20]$. In our two cases the index case had the miliaria skin manifestation, while his younger affected sibling did not show any skin abnormalities. Neither of them had eye problems. In both cases, the disease became milder with age requiring less hospitalization, and their electrolyte status was maintained on oral Sodium Chloride and Sodium Resonium.

\section{Conclusion}

Systemic Type 1 PHA presents as a neonatal emergency and is one of the differential diagnoses of neonatal hyperkalemia and hyponatremia. It can very well be confused with Congenital Adrenal Hyperplasia. The severity and extent of manifestations may differ within the same family. The disease can get milder with advancing age. Genomic testing for the carrier state in the asymptomatic siblings is recommended as well as in the parents to detect homozygosityor compound heterozygosity.

\section{References}

1. Verret F, Hummler E, Schild L and Rossier BC. Control of sodium transport by aldosterone, in The Kidney, Physiology and Physiopathology. Philadelphia: Lippincott Williams and Wilkins. 2000;1441-1471.

2. Riepe FG. Clinical and molecular features of type 1 pseudohypoaldosteronism. Hormone Research. 2009;72(1):1-9. Doi: 10.1159/000224334

3. Zennaro MC, Lombes M. Mineralocorticoid resistance. Trends in Endocrinology and Metabolism. 2004;15(6):264-270.

4. Tajima T, Kitagawa H, Yokoya S, Nakae J, Suwa S, Fujieda K, et al. A novel missense mutation of mineralocorticoid receptor gene in one Japanese family with renal form of pseudohypoaldosteronism type 1. Journal of Clinical Endocrinology and Metabolism. 2000; 85:4690-4694. Doi: 10.1210/jcem.85.12.7078 
5. Saxena A, Hanukoglu L, Saxena D, Thompson RJ, Gardiner RM, Hanukoglu A. Novel mutations responsible for autosomal recessive multisystem pseudohypoaldosteronism and sequence variants in epithelial sodium channel alpha, beta, and gammasubunit genes. Journal of Endocrinology and Metabolism. 2002;87(7):3344-3350. Doi:10.1210/jcem.87.7.8674

6. Amin N, Barth JH, Field HP, Finlay E, Tyerman K, Frazer S, et al. Pseudohypoaldosteronism type 1: clinical features and management in infancy. Endocrine, Diabetes and Metabolism. 2013. Doi: 10.1530/EDM-130010.

7. Rossier BC, Pradervand S, Schild I, Hummler E. Epithelial sodium channel and the control of sodium balance: interaction between genetic and environmental factors. Annals Review of Physiology. 2002;64:877-897. Doi: 10.1146/annurev.physiol.64.082101.143243

8. Attia NA, Marzouk YI. Pseudohypoaldosteronism in a neonate presenting as life-threatening hyperkalemia. Case Reports in Endocrinology. 2016;2016:1-3. doi: 10.1155/2016/6384697.

9. Ranjith G, Uthup S, Satish B, Jain N. Salt wasting disorder in the newborn. Indian Journal of Paediatrics. 2006;73:95-96. Doi: 10.1007/BF02758269

10. Edelheit O, Hanukoglu I, Gizewskat M,Kandemir N,Hanukoglu A, Zajaczek S,et al. Novel mutations in the epithelial sodium channel (ENaC) subunit genes and phenotypic expression of multisystem pseudohypoaldosteronism. Clinical Endocrinology. 2005;62(5):547-553.

11. Silva N, Costa M, Silva A, Carla SA, Sofia M, Olinda $\mathrm{M}$, et al. A case of systemic pseudohypoaldosteronism with a novel mutation in the SCNN1A gene. Endocrinology Nutrition. 2013;60 (1):33-36.

12. Islam MM. The Practice of consanguineous marriage in
Oman: prevalence, trends and determinants. Journal of Biosocial Science. 2012;44(5):571-594.

13. Eaton DC, Helms MN, Koval M, Bao HF, Jain L. The contribution of epithelial sodium channels to alveolar function in health and in disease. Annals Review of Physiology. 2009;71:403-423.

14. Karem E, Bistritzer T, Hanukoglu A, Thomas H, Zhou Z, Bennett W,et al, Pulmonary epithelial sodium channel dysfunction and excess airway liquid in pseudohypoaldosteronism. New England Journal of Medicine. 1999;341:156-162.

15. Urbatsch A, Paller AS. Pustular Miliaria Rubra: a specific cutaneous finding of type 1 pseudohypoaldosteronism. Paediatric Dermatology. 2002;19(4):317-319.

16. Eliwa MS, El-Emmawie AH, Saeed MA. Ocular and Skin manifestations in systemic pseudohypoaldosteronism. British Medical Journal Case Reports. 2014.

17. Nasir A, Najab IA. Unique eyelid manifestations in type 1 pseudohypoaldosteronism. Archives Disease of Childhood Fetal Neonatology. 2012;97(6):F462.

18. Wong GP, Levine D. Congenital Pseudohypoaldosteronism presenting in utero with acute polyhydramnious. Journal of maternal-Fetal medicine. 1998;7:76-78.

19. Akkurt I, Kuhnle U, Ringenberg C. Pseudohypoaldosteronism and cholelithiasis: coincidence or pathogenetic correlation? European Journal of Paediatrics. 1997;156(5):363-366.

20. Saravanapandian N, Paul S, Matthai J. Pseudohypoaldosteronism Type 1: A rare cause of severe dyselectrolytemia and cardiovascular collapse in neonates. Journal of Clinical Neonatology. 2012;1(4):224-226. 